\title{
Bright, guided molecular beam with hydrodynamic enhancement
}

\section{Citation}

Patterson, David, and John M. Doyle. 2007. "Bright, Guided Molecular Beam with Hydrodynamic Enhancement." J. Chem. Phys. 126 (15): 154307. doi:10.1063/1.2717178.

\section{Published Version}

doi:10.1063/1.2717178

\section{Permanent link}

http://nrs.harvard.edu/urn-3:HUL.InstRepos:27868185

\section{Terms of Use}

This article was downloaded from Harvard University's DASH repository, and is made available under the terms and conditions applicable to Other Posted Material, as set forth at http:// nrs.harvard.edu/urn-3:HUL.InstRepos:dash.current.terms-of-use\#LAA

\section{Share Your Story}

The Harvard community has made this article openly available.

Please share how this access benefits you. Submit a story.

Accessibility 


\title{
Bright, guided molecular beam with hydrodynamic enhancement
}

\author{
David Patterson and John M. Doyle ${ }^{\text {a) }}$ \\ Department of Physics, Harvard University, Cambridge, Massachusetts 02138 \\ and Harvard-MIT Center for Ultracold Atoms, Cambridge, Massachusetts 02138
}

(Received 9 February 2007; accepted 22 February 2007; published online 17 April 2007)

\begin{abstract}
The authors realize a novel high flux source of cold atoms and molecules employing hydrodynamic enhancement of an effusive aperture at cryogenic temperatures. Molecular oxygen from the source is coupled to a magnetic guide, delivering a cold, continuous, guided flux of $3 \times 10^{12} \mathrm{O}_{2} \mathrm{~s}^{-1}$. The dynamics of the source are studied by creating and spectroscopically analyzing high flux beams of atomic ytterbium. @ 2007 American Institute of Physics. [DOI: 10.1063/1.2717178]
\end{abstract}

\section{INTRODUCTION}

The study of cold gas phase molecules is a major thrust of modern chemical and atomic physics. ${ }^{1}$ In particular, inelastic collisions of cold molecules have recently attracted widespread theoretical and experimental interest. These collisions are a window into the internal structure and dynamics of molecular interactions as well as being the expected fundamental limiting process for collisional cooling of molecules into and through the ultracold regime. ${ }^{2}$ In addition to collision studies, cold molecules are sought as ideal test objects for precise measurement of fundamental constants [including the search for an electron EDM (Refs. 3 and 4)] and as bits for quantum computing. ${ }^{5,6}$ All of these areas of research benefit from high flux sources of cold molecules and this has spurred widespread interest in realizing such sources.

Although cold atomic sources have been laboratory standards for over a decade, only recently have similar sources of molecules been demonstrated. This is largely because the complex level structure of molecules makes laser coolingthe workhorse of atom cooling - extremely difficult. Demonstrated techniques to produce cold, low velocity samples of molecules are buffer gas cooling, ${ }^{7}$ billiardlike collisions, ${ }^{8}$ filtering slow molecules from an effusive source, ${ }^{9,10}$ deceleration of molecules from a seeded supersonic source in timevarying electric fields, ${ }^{1,11}$ photoassociation of cold and ultracold atoms, ${ }^{12}$ and the formation of molecules from ultracold atoms using Feshbach resonances. ${ }^{13}$ In general, the fluxes achieved with these molecular sources have been far lower than those achieved in cold atom sources.

We earlier ${ }^{14}$ reported a molecular and atomic beam source based on buffer gas cooling, showing that cryogenic cooling of ablated atoms and molecules could effectively produce cold, near-effusive beams. By employing a cold cell $(\approx 4 \mathrm{~K})$ filled with helium gas and several solid ablation targets (e.g., $\mathrm{PbO}$ ), pulsed beams were produced with temperature and forward velocity equivalent to $5 \mathrm{~K}$. This was realized by producing gas phase molecules with short-pulse laser ablation inside the cell. A millimeter size orifice in the side of the cell allowed a small fraction of the cold molecules to

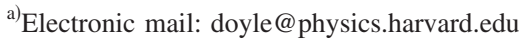

escape into a cryogenic high-vacuum region, forming a beam. The ratio of the number of molecules cooled in the cell to the number emitted into the beam was determined by the ratio of the orifice area to the total area of the interior of the cell, about $10^{-3}$. It was estimated that beams of molecules from this source coupled to a magnetic or electric guide would produce a peak guided flux not higher than $10^{9}$ molecules s ${ }^{-1}$.

In this letter we realize a cold molecule buffer gas source that uses hydrodynamic effects to vastly enhance the molecular beam flux compared to the old source. Oxygen is used as our test molecule. We show that this new source can successfully load a magnetic guide, producing a guided beam of cold $\mathrm{O}_{2}$ with a continuous flux of $3 \times 10^{12}$ molecules s ${ }^{-1}$ into a room-temperature high-vacuum region. This is 1000 times greater than that predicted with the old source. This flux compares favorably to cold atom sources and far exceeds previous demonstrated fluxes of cold molecules. Using atomic ytterbium, we further characterize the creation and dynamics of the beam before it enters the guide. We develop a simple model of hydrodynamic enhancement within the cell and find reasonable agreement between this model and our data. The performance of this guide/source combination is well described by Monte Carlo simulation.

\section{BEAM APPARATUS}

The heart of our experimental apparatus is an $\approx 2.5 \mathrm{~cm}$ size cell anchored to the cold plate of a cryostat. This cell is a copper box with two fill lines (one for helium and one for oxygen) on one side, an aperture on the opposite side, an $\mathrm{Yb}$ ablation target inside, and windows for laser access. In these experiments we create beams of either atomic $\mathrm{Yb}$ or $\mathrm{O}_{2}$. To produce beams of $\mathrm{O}_{2}$ we flow $\mathrm{He}$ and $\mathrm{O}_{2}$ continuously into the cell where they mix and thermalize to the temperature of the cell. The He does not freeze to the walls of the cell but any $\mathrm{O}_{2}$ molecule that touches the wall does. The $\mathrm{O}_{2}$ cools by thermalizing with the helium. The helium and a fraction of the $\mathrm{O}_{2}$ escape out through a double-stage aperture (see Sec. $3 \mathrm{~A}$ ), forming a beam, with the balance of the $\mathrm{O}_{2}$ remaining frozen on the cell walls. The same general processes of thermalization and beam creation occur with $\mathrm{Yb}$. To produce 

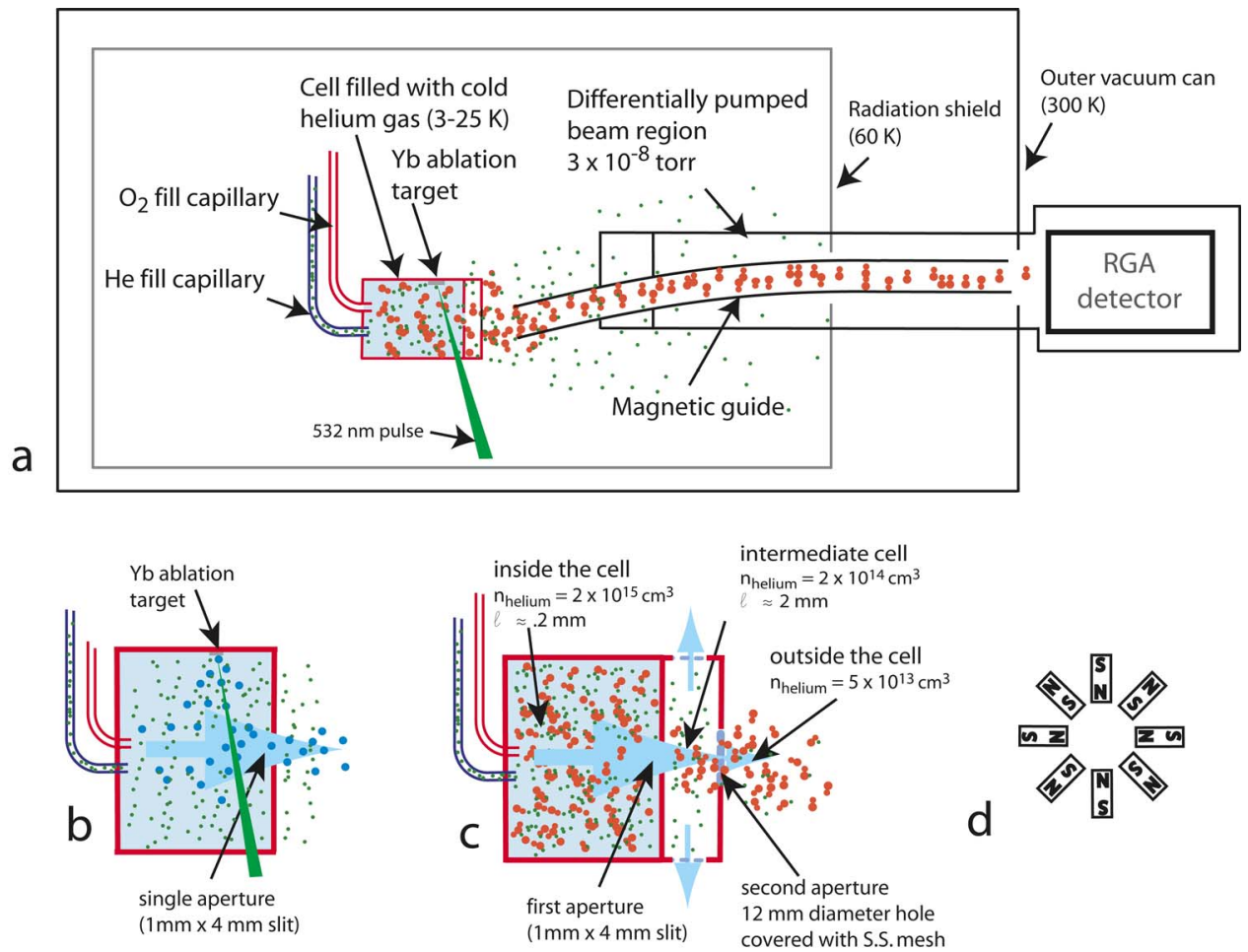

FIG. 1. (Color online) Experimental apparatus. (a) Hot $(\approx 100 \mathrm{~K}) \mathrm{O}_{2}$ molecules or ablated $\mathrm{Yb}$ atoms thermalize with helium gas in a cold (3-25 K) copper cell. The mixture then sprays through the slit aperture and enters a beam guide. Cold, low field seeking molecules are guided into a RGA detector. (b) "One-stage aperture" detail. This aperture was characterized with laserablated atomic Yb. The mean free path in the main cell (left) is generally short enough to thermalize hot $\mathrm{Yb}$ atoms before they touch and stick to the wall of the cell. The slit aperture allows this mixture to form a beam in the Dewar vacuum. Hydrodynamic entrainment greatly enhances the flow through this aperture compared to a simple effusive source. This configuration produced high flux atomic Yb beams with significant forward velocity (see Fig. 3 and discussion on page 6). (c) "Two-stage aperture" detail. This two-stage aperture is implemented with the idea to produce a high flux effusive source. As in (b), a mixture of $\mathrm{He}$ and $\mathrm{O}_{2}$ first exits from the cell through a slit aperture. However, in this case, rather than enter the beam directly, the mixture enters a small second cell. Additional venting holes on the side of the second cell maintain a low enough pressure that flow through the final aperture is largely effusive. The $\mathrm{He}$ density in the second chamber is much lower than in the first cell, so $\mathrm{O}_{2}$ molecules experience just a few more collisions before being diffused through the final aperture. These collisions are sufficient to rethermalize the molecules that have been hydrodynamically boosted due to their passage through the first aperture. (d) Magnet detail. The magnetic guide is an octopole, composed of eight $\mathrm{NdFeB}$ permanent magnets, and has a depth of $\approx 5000 \mathrm{G}\left(680 \mathrm{mK}\right.$ for $\left.\mathrm{O}_{2}\right)$. Low field seekers are confined to the center of the guide.

beams of $\mathrm{Yb}$ we flow He continuously into the cell and ablate the $\mathrm{Yb}$ target with a pulsed laser $(4 \mathrm{~ns}, 532 \mathrm{~nm}, 10 \mathrm{~mJ})$.

In more detail, the copper cell is $2.5 \times 2.5 \times 2.5 \mathrm{~cm}^{3}$ $\left(V \approx 15 \mathrm{~cm}^{3}\right)$ and its temperature is varied between $T=2.6$ and $25 \mathrm{~K}$. It is surrounded by (Dewar) vacuum, heat shields, and optical windows [see Fig. 1(a)]. Cooling of the cell is done with a pulsed tube refrigerator and a small pumped liquid helium reservoir (used to achieve temperatures below $4 \mathrm{~K}$ ). The copper He gas fill line to the cell is thermally anchored to a $1.3 \mathrm{~K}$ cold plate a few centimeters before it enters the cell. The $\mathrm{O}_{2}$ fill line is held at $100 \mathrm{~K}$, above the freezing point of oxygen. A short stainless steel sleeve thermally disconnects the relatively warm $\mathrm{O}_{2}$ line from the cell. Helium is typically flowed into the cell at $1 \times 10^{17}-8$ $\times 10^{18}$ at. $\mathrm{s}^{-1}$ and $\mathrm{O}_{2}$ is flowed into the cell at similar rates, typically $1 \times 10^{17}-3 \times 10^{18}$ molecules $\mathrm{s}^{-1}$.

Despite the large He gas flow, the beam region vacuum is maintained at a low $3 \times 10^{-8}$ torr by two stages of differential pumping with high speed cryopumps made of activated charcoal ( $T=6.5 \mathrm{~K}, 2000 \mathrm{~cm}^{2}$ total apparent area). A $30 \mathrm{~cm}$ long magnetic guide [see Fig. 1(d)] can be placed with the guide entrance $1 \mathrm{~cm}$ from the aperture. The guide is constructed from an array of permanent $\mathrm{NdFeB}$ magnets (each magnet $0.3 \times 0.6 \times 7.5 \mathrm{~cm}^{3}$ ). The magnets are arranged in a linear octopole, such that low field seeking molecules are confined to the center of the guide. The far end of the guide (the "guide exit") is not at cryogenic temperatures, but rather deposits the guided molecules into a room-temperature residual gas analyzer (RGA). The guide has an inner diameter of $0.9 \mathrm{~cm}$ and $5000 \mathrm{G}$ depth, equivalent to $680 \mathrm{mK}$ for $\mathrm{O}_{2}$ in its most magnetic guidable state. The guide undergoes a single bend with radius of curvature $r=20 \mathrm{~cm}$. This bend ensures that only guided molecules can pass; i.e., $\mathrm{O}_{2}$ must be moving slower than $7000 \mathrm{~cm} \mathrm{~s}^{-1}$ ( $9 \mathrm{~K}$ equivalent temperature) to be guided around the bend. The entire system imposes a heat load of $\approx 1 \mathrm{~W}$ on the $4 \mathrm{~K}$ refrigerator.

\section{RESULTS}

We run two different types of experiments, guided (with $\mathrm{O}_{2}$ ) and unguided (with $\mathrm{Yb}$ ). In the guided work, we beam $\mathrm{O}_{2}$ into a magnetic guide and measure with the RGA the flux of $\mathrm{O}_{2}$ exiting the guide. The RGA is calibrated by comparison to a NIST-traceable standard $\mathrm{O}_{2}$ leak. In the unguided work, we remove the guide assembly and use $\mathrm{Yb}$ to characterize the beam source. The flux and velocity profile of the $\mathrm{Yb}$ beam and the density and temperature of $\mathrm{Yb}$ gas in the cell are all measured using laser absorption spectroscopy. 


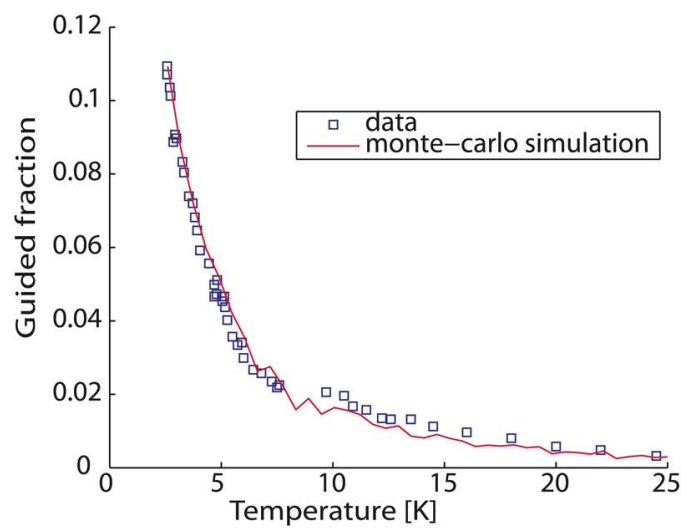

FIG. 2. (Color online) Guided flux of $\mathrm{O}_{2}$ as a function of cell temperature. The temperature dependence of the measured flux is in excellent agreement with the Monte Carlo simulation (solid line). The vertical axis represents the absolute guided fraction (the ratio of the number of guided $\mathrm{O}_{2}$ molecules to the number exiting the source) as calculated by the simulation. The only free parameter in this fit is the absolute scale. In order to run for extended periods of time while avoiding plugging the aperture, these data were taken with oxygen input (0.5 SCCM) and lower helium input (0.4 SCCM) about one order of magnitude lower than that used to demonstrate our peak flux of $3 \times 10^{12} \mathrm{O}_{2} \mathrm{~s}^{-1}$. The highest flux shown here represents $2.5 \times 10^{11} \mathrm{O}_{2} \mathrm{~s}^{-1}$.

We chose to guide $\mathrm{O}_{2}$ because it is relatively easy to introduce into our cold cell and has a high magnetic moment of $2 \mu_{B}$. Because the $\mathrm{O}_{2}$ is cooled in zero magnetic field, it is expected that the molecules occupy a uniform statistical mixture of Zeeman states. Thus, $\approx 1 / 9$ of the molecules exiting the aperture are in the maximally guided $N=1, J=2, m_{J}=2$ state, where $N$ is the rotational quantum number, $J$ is the total angular momentum quantum number, and $m_{J}$ is its projection on the axis defined by the magnetic field. We estimate that the contribution to guided beam flux from other states is small $(<10 \%)$.

The molecule source and guide were characterized by varying the $\mathrm{O}_{2}$ flow, $\mathrm{He}$ flow, and cell temperature, while always monitoring the output flux of $\mathrm{O}_{2}$. Figure 2 shows our guided flux for a range of temperatures. Because the depth of the guide $(680 \mathrm{mK})$ is less than the temperature of the source over the entire range of temperatures used, a colder source will lead to a higher flux of guided molecules. This is seen in the data. A Monte Carlo simulation of our guide is plotted with the data. Agreement is excellent, showing not only that we are guiding molecules but that the details of the system are well understood.

Our maximum measured guided flux of $3 \times 10^{12} \mathrm{O}_{2} \mathrm{~s}^{-1}$ is achieved by simultaneously flowing $10 \mathrm{SCCM}$ of $\mathrm{O}_{2}$ and $2 \mathrm{SCCM}$ of He into the cell (SCCM denotes cubic centimeter per minute at STP). The system can operate at this peak flux for about $100 \mathrm{~s}$ before the aperture becomes plugged with oxygen ice.

We note that we are confident that the $\mathrm{O}_{2}$ in our guide is cold rotationally as well as translationally. In all our previous work with buffer gas cooling and in many supersonic beam experiments, it has been observed that rotational thermalization rates are comparable or faster than elastic scattering rates. ${ }^{7,15}$

The high guided fluxes of $\mathrm{O}_{2}$ we achieve are due to a newly utilized effect in our beam source, hydrodynamic en- hancement. We first describe a simple theory of this effect and then describe experiments with $\mathrm{Yb}$ that verify this basic picture.

The helium gas in our cell can be characterized by mean free path $\ell$ (typically, $\ell \approx 0.1 \mathrm{~cm}$ ). Atoms and molecules can leave the cell via two processes: by diffusing to the cell wall (with a small fraction of the molecules diffusing out of the aperture) and by being entrained in the helium flow that is continuously leaving through the aperture. If the diffusion time for an atom or molecule $\left(\tau_{\text {diffusion }}\right)$ is much shorter than the characteristic time a helium atom (or entrained species) spends in the cell $\left(\tau_{\text {pumpout }}\right)$, hydrodynamic effects can be ignored. In this diffusive limit, the fraction that escape into the beam from a cell of side length $L$ with aperture area $A$ is $f \approx \pi L^{2} / A$ (about about $2 \times 10^{-3}$ for our cell). If, on the other hand, $\tau_{\text {diffusion }} \gg \tau_{\text {pumpout }}$, a large fraction are entrained inside the cell and are forced to move toward the aperture and eventually into the beam. The dimensionless parameter

$$
\gamma \equiv \frac{\tau_{\text {diffusion }}}{\tau_{\text {pumpout }}}
$$

therefore provides a good indication of which limit the cell is in, with $\gamma \ll 1$ means diffusion limit and $\gamma \gg 1$ means entrainment limit. We note that the entrainment being discussed here is not the same effect that is seen in typical supersonic nozzles, where collisions within the nozzle (as opposed to the volume well before the nozzle) are important and flow velocities are large compared to the sonic velocity. The mean flow velocity of He within our cell is typically much less than the speed of sound in the He gas.

Exact analysis depends on geometrical details, but it can be easily shown that $\gamma=k A / 4 \ell L$, with $k$ a dimensionless constant of order unity. Ideally one wishes to operate with as large a value of $\gamma$ (large helium density) as possible while maintaining an effusive source. If the density of helium just outside the aperture is large enough that the mean free path is smaller than the aperture size, then entrainment outside the aperture results in a significant increase in the mean forward velocity (i.e., "forward boost") of the beam. This is a detriment to guiding experiments as the boost makes a smaller fraction of the total beam flux guidable.

Experimental study of these processes is needed to verify our model. As $\mathrm{O}_{2}$ is very difficult to detect optically, we chose to characterize the flux and velocity distribution of the beam with atomic ytterbium, which has easily accessible optical transitions.

Two aperture configurations were demonstrated. Figure 3 shows the output efficiency of a "one-stage aperture" [see Fig. 1(b)] consisting of a simple slit aperture $\left(1 \times 4 \mathrm{~mm}^{2}\right)$, where this efficiency is defined as the ratio of the number of $\mathrm{Yb}$ in the beam to the number of cold $\mathrm{Yb}$ produced by ablation in the cell. At buffer gas flows where $\gamma>1$ (high flows), up to $40 \%$ of the cold $\mathrm{Yb}$ atoms in the cell are detected in the resultant beam. The divergence of the beam is measured by comparing the Doppler shifts in the absorption spectra parallel and transverse to the atomic beam. The beam is somewhat collimated compared to a pure effusive source, 


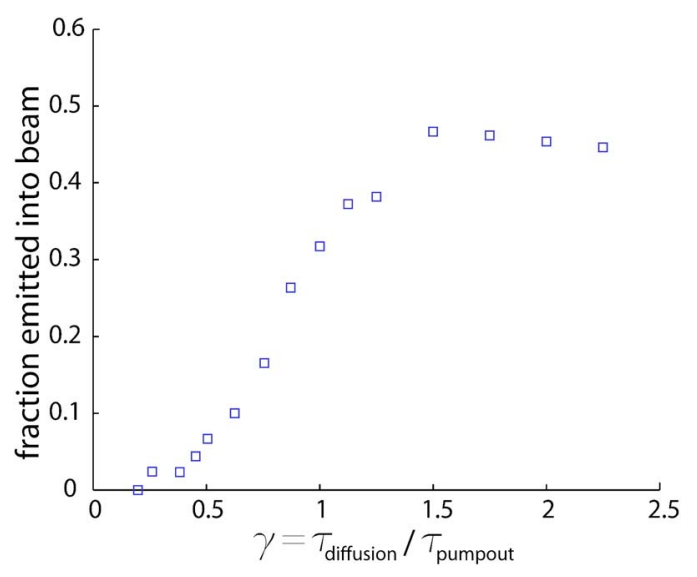

FIG. 3. (Color online) Plotted is the ratio of the number of ablated and cooled ytterbium atoms to the number emitted into the beam for a singlestage aperture. At high buffer gas densities, which correspond to long diffusion times and therefore high $\gamma$ (see main text), up to $40 \%$ of the cold ablated atoms are detected in the beam. The peak instantaneous flux represented here is $5 \times 10^{15}$ at. $\mathrm{s}^{-1} \mathrm{sr}^{-1}$.

with a divergence of $\approx 0.1 \mathrm{sr}$. The peak flux of 6 $\times 10^{15}$ at. $\mathrm{s}^{-1} \mathrm{sr}^{-1}\left(5 \times 10^{12}\right.$ at./pulse $)$ suggests that this simple cryogenic one-stage aperture provides an attractive alternative to seeded supersonic jet sources of cold molecules. ${ }^{16}$ For trapping, where total kinetic energy must be less than the trap depth, typically a few Kelvins, this beam's high forward boost (velocity $=130 \mathrm{~m} \mathrm{~s}^{-1} \approx 160 \mathrm{~K}$ effective temperature) makes it unsuitable.

Trapping work would ideally use a beam with both high flux and minimal forward boost. In an attempt to achieve this best of both worlds, the "two-stage aperture" shown in Fig. 1(c) was developed. In this two-stage aperture the $\mathrm{He}-\mathrm{Yb}$ mixture still passes through the $1 \times 4 \mathrm{~mm}^{2}$ slit with a large hydrodynamic flux enhancement and corresponding boost. But, instead of directly entering the vacuum beam region, this boosted beam enters a small chamber. Atoms can pass from the small chamber through a $12 \mathrm{~mm}$ diameter aperture on the opposite side. This second, larger aperture is covered by a stainless steel mesh with a pore size of $140 \mu \mathrm{m}(28 \%$ transparency) and creates a near-effusive beam in the vacuum region. The He density in the second chamber would be far too low to thermalize hot atoms entering the cell. However, it is high enough to thermalize the cold (but boosted) $\mathrm{Yb}$ that comes through the first orifice and low enough so that $\gamma \ll 1$, i.e., diffusion dominated dynamics. The final aperture is large enough to pass into the beam about $4 \%$ of atoms that arrive in the second chamber. Finally, it is hypothesized that the small pores in the mesh may help reduce hydrodynamic, noneffusive flow inside and just outside the nozzle. Preliminary results in our laboratory with and without the mesh provide some evidence that this is the case.

The $\mathrm{Yb}$ beam from the two-stage aperture has a mean velocity of $35 \mathrm{~m} \mathrm{~s}^{-1}$, with a spread of $20 \mathrm{~m} \mathrm{~s}^{-1}$. The total beam flux of $5 \times 10^{10}$ atoms for each $\mathrm{Yb}$ ablation pulse represents about $1 \%$ of the cold atomic $\mathrm{Yb}$ produced in the ablation, or about $3 \%$ of the output of the one-stage aperture. In our guided $\mathrm{O}_{2}$ experiments, the two-stage aperture is used.

\section{POTENTIAL APPLICATIONS}

The one-stage aperture cold source described above can provide high fluxes of any atom or molecule. For example, under the assumption of $10^{12}$ cooled radicals of $\mathrm{CaF}$ per ablation pulse ${ }^{17}$ and the use of a $15 \mathrm{~Hz}$ pulsed yttrium aluminum garnet laser, a $15 \%$ duty cycle pulsed beam would be produced with a continuous flux of 7 $\times 10^{13}$ molecules s${ }^{-1} \mathrm{sr}^{-1}$ (10 ms pulse length, $0.1 \mathrm{sr}$ divergence, peak flux $5 \times 10^{14}$ molecules $\mathrm{s}^{-1} \mathrm{sr}^{-1}$ ). These fluxes are 500-1000 times higher than typical fluxes from seeded supersonic beams of radicals at comparable translational and rotational temperatures. ${ }^{16}$ As described above, we produce even brighter cold atomic beams, with a continuous flux of $7 \times 10^{14}$ at. $\mathrm{s}^{-1} \mathrm{sr}^{-1}$ (peak flux $5 \times 10^{15}$ at. $\mathrm{s}^{-1} \mathrm{sr}^{-1}$ ). This source is therefore an attractive option for collisional studies and high precision measurements based on cold molecules as well as atoms. 4,18

The two-stage aperture source could be applied to trapping polar molecules. Observing collisions in trapped samples of polar molecules has proven to be difficult, primarily because available fluxes of cold polar molecules are typically low. Replacing the magnetic guide demonstrated here with a simple electrostatic guide (as in Refs. 9 and 19) could realize a guided source of polar molecules with a phase space density 300 times higher than has been demonstrated to date. We note that the trap lifetimes for polar molecules in a $300 \mathrm{~K}$ environment are limited to a few seconds by optical pumping via thermal microwaves, a constraint which is conveniently avoided by our cryogenic environment. ${ }^{20}$

Although in these experiments atoms and molecules were introduced into the cell using ablation and gaseous flow, direct loading from a molecular beam (e.g., oven or pulsed discharge) is also possible. ${ }^{21,22}$ Thus, this source is truly general, being able to cool molecules produced by any technology.

\section{CONCLUSION}

We have demonstrated production of cold, guided $\mathrm{O}_{2}$ with a flux of $3 \times 10^{12} \mathrm{O}_{2} \mathrm{~s}^{-1}$. We have further demonstrated a general long-pulse source of cold, unguided atoms or molecules with a peak flux of $5 \times 10^{14} \mathrm{Yb} \mathrm{s}^{-1}$. The efficiency of the unguided source can be near unity, with the number atoms or molecules in the cold beam being up to $40 \%$ of the number cooled in the cell.

\section{ACKNOWLEDGMENTS}

The authors are grateful for the contributions of Y. Takahashi and group members by introducing them to $\mathrm{Yb}$ for use in beam experiments. This work was funded by NSF Grant No. PHY-0457047.

\footnotetext{
${ }^{1}$ H. L. Bethlem and G. Meijer, Int. Rev. Phys. Chem. 22, 73128 (2003).

${ }^{2}$ J. Bohn, Phys. Rev. A 63, 052714 (2001).

${ }^{3}$ M. G. Kozlov and L. N. Labzowsky, J. Phys. B 28, 1933 (1995); URL: http://stacks.iop.org/0953-4075/28/1933

${ }^{4}$ J. Hudson, B. Sauer, M. Tarbutt, and E. A. Hinds, Phys. Rev. Lett. 89, 023003 (2002).

${ }^{5}$ D. DeMille, Phys. Rev. Lett. 88, 067901 (2002).

${ }^{6}$ P. Rabl, D. DeMille, J. M. Doyle, M. D. Lukin, R. J. Schoelkopf, and P.
} 
Zoller, Phys. Rev. Lett. 97, 033003 (2006).

${ }^{7}$ J. D. Weinstein, R. deCarvalho, T. Guillet, B. Friedrich, and J. M. Doyle, Nature (London) 395, 148 (1998).

${ }^{8}$ M. S. Elioff, J. J. Valentini, and D. W. Chandler, Science 302, 1940 (2003).

${ }^{9}$ S. A. Rangwala, T. Junglen, T. Riegen, P. W. H. Pinhse, and G. Rempe, Phys. Rev. A 67, 043406 (2003).

${ }^{10}$ E. Nikitin, E. Dashevskaya, J. Alnis, M. Auzinsh, E. R. I. Abraham, B. R. Furneaux, M. Keil, C. McRaven, N. Shafer-Ray, and R. Waskowsky, Phys. Rev. A 68, 023403 (2003).

${ }^{11}$ H. L. Bethlem, G. Berden, and G. Meijer, Phys. Rev. Lett. 83, 1558 (1999).

${ }^{12}$ J. M. Sage, S. Sainis, T. Bergeman, and D. DeMille, Phys. Rev. Lett. 94, 203001 (2005).

${ }^{13}$ S. T. T. Elizabeth, A. Donley, N. R. Claussen, and C. E. Wieman, Nature (London) 417, 529 (2001).

${ }^{14}$ S. E. Maxwell, N. Brahms, R. deCarvalho, D. R. Glenn, J. S. Helton, S. V. Nguyen, D. Patterson, J. Petricka, D. DeMille, and J. M. Doyle, Phys. Rev. Lett. 95, 173201 (2005).
${ }^{15}$ J. M. Hollis and D. Phillips, Jet Spectroscopy and Molecular Dynamics (Springer, Berlin, 2004), p. 74.

${ }^{16}$ M. R. Tarbutt, J. J. Hudson, B. E. Sauer, E. A. Hinds, V. A. Ryzhov, V. L. Ryabov, and V. F. Ezhov, J. Phys. B 35, 5013 (2002).

${ }^{17}$ D. Egorov, J. Weinstein, D. Patterson, B. Friedrich, and J. Doyle, Phys. Rev. A 63, 030501 (2001).

${ }^{18}$ D. Budker, in Physics Beyond the Standard Model, Proceedings of the Fifth International WEIN Symposium, edited by P. Herczeg, C. M. Hoffman, and H. V. Klapdor-Kleingrothaus (World Scientific, Singapore, 1999), pp. 418-441.

${ }^{19}$ H. L. Bethlem, G. Berden, F. M. H. Crompvoets, R. T. Jongma, A. J. A. van Roij, and G. Meijer, Nature (London) 406, 491 (2000).

${ }^{20}$ S. Hoekstra, J. J. Gilijamse, B. Sartakov, N. Vanhaecke, L. Scharfenberg, S. Y. T. van de Meerakker, and G. Meijer, Phys. Rev. Lett. 98, 133001 (2007).

${ }^{21}$ D. Egorov, W. Campbell, B. Friedrich, S. Maxwell, E. Tsikata, L. vanBuuren, and J. Doyle, Eur. Phys. J. D 31, 307 (2004).

${ }^{22}$ W. C. Campbell, E. Tsikata, H.-I. Lu, and J. M. Doyle, Phys. Rev. Lett. (to be published).. 